Projets

de paysage

\section{Projets de paysage}

Revue scientifique sur la conception et l'aménagement de l'espace

$16 \mid 2017$

Arbres et paysages

\title{
Les enjeux du végétal dans une ville du «Sud »
}

Le cas de la ville de Marrakech et la fin d'un modèle de " cité-jardin » The Importance of Plant Life in a City of the "South" - The Case of Marrakesh and the End of "Garden-City" Model

Mustapha El Hannani, Aude Nuscia Taïbi, Naïma Brabra et Sigrid Giffon

\section{CpenEdition}

\section{Journals}

Édition électronique

URL : http://journals.openedition.org/paysage/5682

DOI : 10.4000/paysage.5682

ISSN : 1969-6124

\section{Éditeur :}

École nationale supérieure du paysage de Versailles-Marseille, Institut national des sciences appliquées Centre Val de Loire - École de la nature et du paysage, École nationale supérieure d'architecture et de paysage de Bordeaux, École nationale supérieure d'architecture et de paysage de Lille, Agrocampus Angers

Référence électronique

Mustapha El Hannani, Aude Nuscia Taïbi, Naïma Brabra et Sigrid Giffon, «Les enjeux du végétal dans une ville du « Sud » », Projets de paysage [En ligne], 16 | 2017, mis en ligne le 02 juillet 2017, consulté le 30 avril 2020. URL : http://journals.openedition.org/paysage/5682 ; DOI : https://doi.org/10.4000/ paysage. 5682

Ce document a été généré automatiquement le 30 avril 2020.

Projets de paysage 


\title{
Les enjeux du végétal dans une ville $\mathrm{du} \ll \mathrm{Sud} »$
}

\author{
Le cas de la ville de Marrakech et la fin d'un modèle de « cité-jardin » \\ The Importance of Plant Life in a City of the "South" - The Case of Marrakesh \\ and the End of "Garden-City" Model
}

Mustapha El Hannani, Aude Nuscia Taïbi, Naïma Brabra et Sigrid Giffon

1 L'enveloppe végétale de la ville de Marrakech dominée par des palmiers et arbres fruitiers est le premier contact visuel qu'offre la ville aux nombreux voyageurs arabes ou occidentaux qui décrivent une ville noyée dans un écrin de verdure contrastant avec son environnement à caractère " désertique ».

2 Cette ville impériale est l'une des plus grandes cités amazigho-arabes de l'occident musulman. Édifiée par Youssef Ibn Tachfine en 1062, elle s'est développée sur le modèle classique de la cité-jardin (El Faïz, 1996 et 2000) sans s'inscrire dans aucune continuité historique en lien avec une cité antique. Cette cité-jardin s'insère dans une oasis, la seule dans cette région au nord du Haut Atlas. L'origine et l'histoire de cette enveloppe végétale, la palmeraie, paysage artificiel, sont encore très mal connues (Aârabi, 2014). Pourtant la palmeraie est indissociable de la ville de Marrakech dans la plupart de ses représentations iconographiques et de ses descriptions d'écrivains et de voyageurs (Chevrillon, 2002; Tharaud, 2012). La ville de Marrakech, première destination touristique du Maroc, a su mettre en avant dès la période coloniale son cadre paysager, associant le minéral (bâti, jbel lkhdar, rocher dominant la ville nouvelle, « Médina ») et le végétal, dont l'élément identitaire le plus fort est la palmeraie. Cette image, qui perdure depuis la période coloniale, est de plus en plus déconnectée de la réalité, la cité-jardin et l'oasis s'estompant au fur et à mesure de l'extension et de la densification de la ville dès la période coloniale et depuis l'indépendance du pays. Cette croissance urbaine, particulièrement accélérée depuis les années 1990, se fait désormais "à l'aveugle ", aucun plan d'urbanisme de l'agglomération n'ayant été respecté et le nouveau plan étant déjà obsolète. Elle se traduit par l'accentuation du caractère minéral de la ville, le végétal ayant tendance à reculer dans l'espace public où il n'est 
souvent plus représenté que par quelques arbres alignés le long de certaines voies de communication et des jardins, peu nombreux, souvent mal entretenus à l'exception d'un petit nombre, emblématiques et restaurés (El Harti, Aarsat Moulay Abdeslam, Ménara). Dans l'espace public fréquenté par les touristes, à proximité des lieux touristiques, le végétal reste en revanche bien représenté (Boujrouf, 2005) ainsi que dans certains quartiers de villas. Dans ces quartiers huppés, le végétal public se double de jardins privés caractérisés par une végétation luxuriante qui génère des ambiances qui rappellent celles décrites dans les cités-jardins avec leur foisonnement végétal. Ces deux composantes expliquent probablement la persistance de cet imaginaire chez les touristes et dans l'iconographie, support de communication à leur intention.

La palette végétal (arbres, arbustes, plantes ornementales, etc.), utilisée dans ces lieux, révèle un changement qualitatif, observé sur le terrain, dans l'évolution des paysages végétaux de la cité. Elle est aussi le reflet de la classe sociale et des populations dont les goûts et les choix remettent en cause «l'identité» de la ville ocre, basée historiquement sur un subtil équilibre entre le végétal et le minéral comme nous le verrons par la suite.

C'est dans ce contexte que la question de la place du végétal sous toutes ses formes (public, privé, jardins, parcs, espaces agricoles urbains, trame verte, etc.) doit être reposée à l'échelle de cette cité. Si la ville bénéficie toujours d'une image de "ville verte », comme l'illustre l'iconographie à destination des touristes nationaux et surtout internationaux et des projets immobiliers, le constat est là : en 25 ans, $80 \%$ des espaces verts intra-muros ont disparu (El Faïz, 2002), instaurant un déséquilibre irréversible entre minéral et végétal, et constituant une double rupture entre deux modèles, celui de la médina qui a perduré plus de dix siècles et celui de la ville coloniale.

Notre étude montre que ce sont également les fonctions de ce végétal, notamment public, qui ont profondément évolué ces dernières décennies, avec une focalisation sur les fonctions paysagères et récréatives, et une disparition progressive des fonctions environnementales et économiques. Ainsi, la palmeraie est devenue un simple décor, de nombreux quartiers sont dépourvus de végétation, les jardins publics son rares et, là où le végétal se développe, il est associé à des lieux dédiés au tourisme ou habités par une population aisée.

6 L'analyse de la dynamique et de la place du végétal à travers ses différentes formes et fonctions à l'échelle de trois modèles, la médina, la ville coloniale et les nouveaux quartiers, permet de mieux comprendre les enjeux qu'il représente et la place qu'il occupe dans la fabrication d'une cité jadis qualifiée d'oasis et aujourd'hui devenue presque minérale. S'agit-il d'un processus irréversible révélateur d'une rupture d'ordre culturel dans la fabrication de la ville?

\section{Une typologie et une spatialisation du végétal par le terrain et la télédétection}

7 La zone d'étude analysée choisie correspond à l'espace urbain et périurbain de Marrakech et ne coïncide pas avec les limites administratives de la ville (figure 1). Les limites de la zone dépassent parfois celles de la commune urbaine pour intégrer de nouveaux quartiers, à l'ouest notamment, et sont moins étendues que celle-ci pour exclure des zones trop « rurales », notamment à l'est (la palmeraie). La limite nord est 
marquée par l'oued Tensift, la limite sud par le rempart du jardin d'Agdal. In fine, la superficie de la zone d'étude est de 159,39 km² (15 939 hectares).

Figure 1. Limites de la zone d'étude et quartiers administratifs de Marrakech

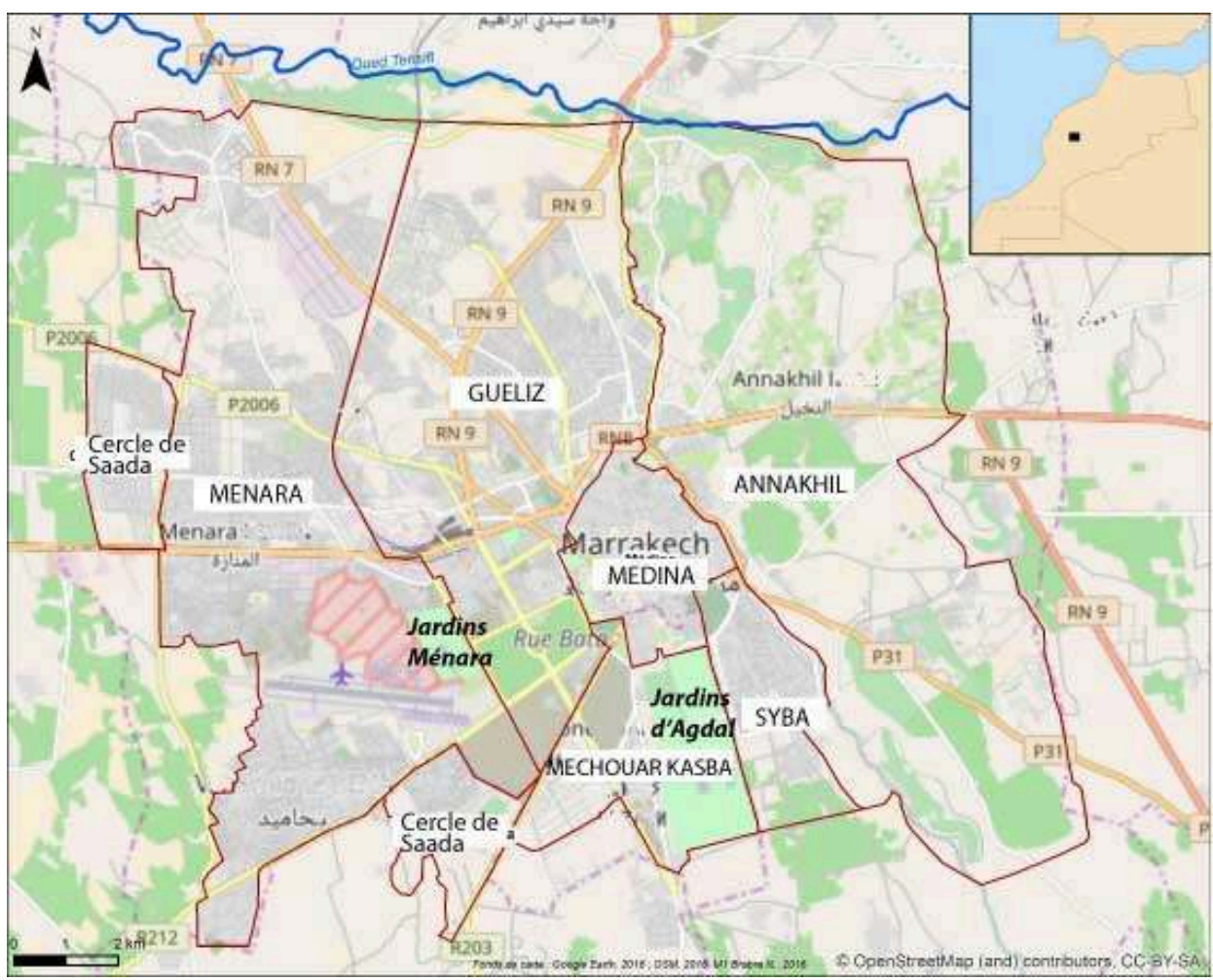

L'analyse qualitative et quantitative du végétal a été réalisée à l'échelle des quartiers. Cependant, là encore, les limites administratives ne sont pas adaptées à ce sujet car elles n'intègrent pas de dimension historique. Nous avons donc travaillé à partir de limites de quartiers définies sur la base de leur morphologie et/ou de leurs fonctions (figure 2). 


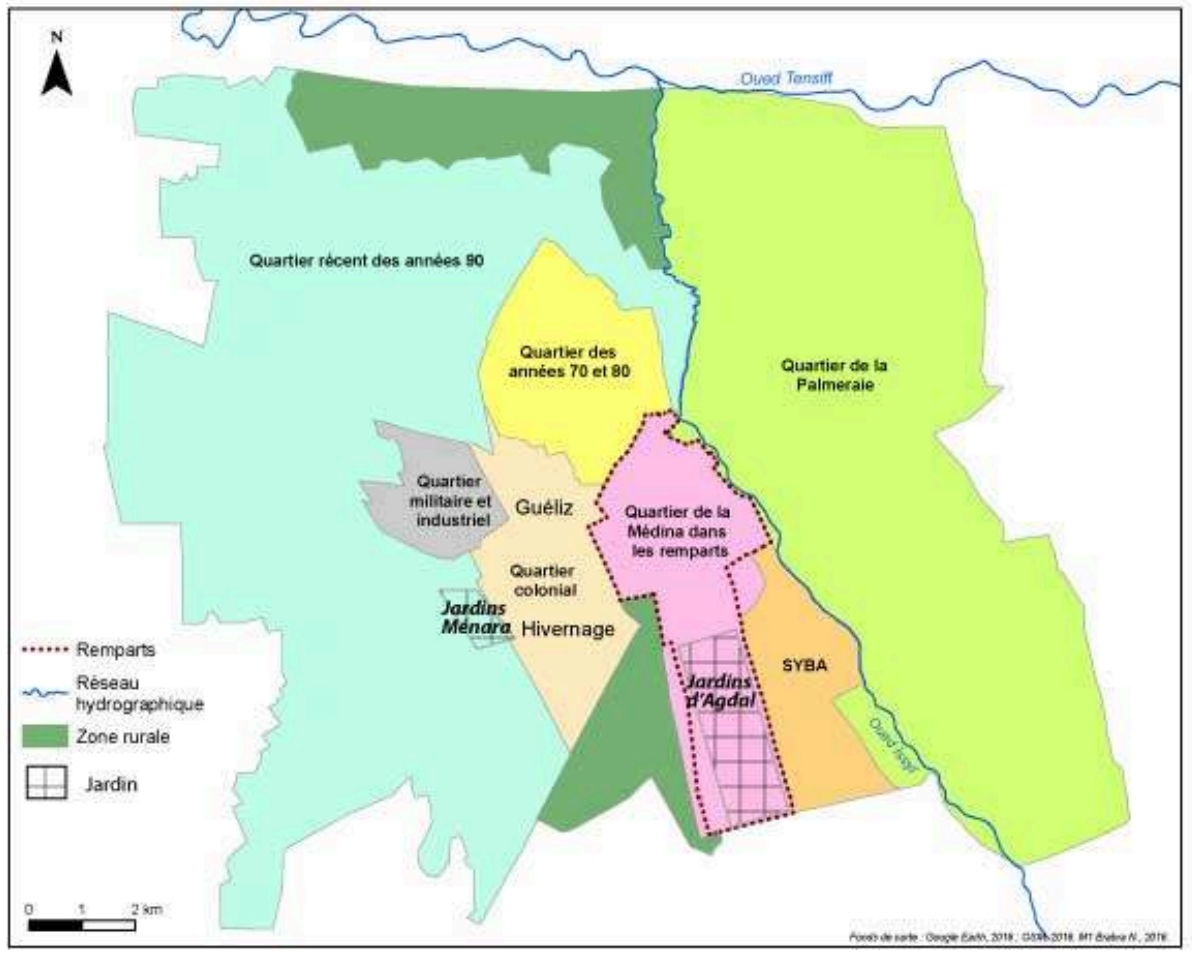

9 Ainsi, concernant le quartier de la médina, nous avons travaillé successivement en incluant ou en excluant les jardins d'Agdal, aujourd'hui administrativement rattachés à Mechouar-Kasbah (figures 1 et 2), qui se localisent à l'intérieur des remparts mais augmentent artificiellement le degré de végétalisation de la médina, à dominante minérale. Les remparts nous ont également servi de limites pour certaines quantifications alors qu'ils ne coïncident pas avec les limites administratives des quartiers. Nous avons également distingué le quartier colonial (incluant Guéliz et l'Hivernage), les quartiers construits dans les années 1970 et 1980 au nord de la ville, le quartier militaire et industriel et les quartiers récents construits depuis les années 1990, qui sont intégrés administrativement dans les quartiers de Guéliz et Ménara pour l'essentiel (figures 1 et 2). Seul le quartier Annakhil correspond à celui de la palmeraie, et dans une certaine mesure celui de Sidi Youssef Ben Ali (SYBA) à l'exception d'une petite partie au nord.

Le végétal à Marrakech est de manière générale mal connu. Les services des espaces verts de la ville ne disposent pas de données quantifiées ni cartographiques précises sur le végétal (superficies des espaces verts, types d'espèces, nombre d'arbres d'alignement, etc.). Face à ces lacunes importantes et à un travail de recensement exhaustif considérable, difficilement envisageable, nous avons privilégié une approche à dominante qualitative, secondairement quantifiée, associant images satellites, cartes, documents historiques et archives à de nombreuses missions sur le terrain d'observation et de validation; ceci afin de définir une typologie du végétal et spatialiser sa répartition à différentes périodes (précoloniale, coloniale et postcoloniale jusqu'à la période actuelle). La typologie se base sur des critères de structure d'organisation du végétal, de densité, de surfaces couvertes, d'espèces et de fonctions. Ces éléments ont été identifiés à partir de la photo-interprétation des images Google 
Earth de 2016 et adaptés au fur et à mesure des observations de terrain et de la fréquentation des différents quartiers de la ville. Nous nous sommes également appuyés sur des éléments de morphologie urbaine pour affiner la typologie. Cette méthodologie a permis d'identifier 18 classes en 2016, qui ont été regroupées en quatre grands ensembles subdivisés en sous-ensembles (figure 3).

Figure 3. Typologie du végétal à Marrakech en 2016

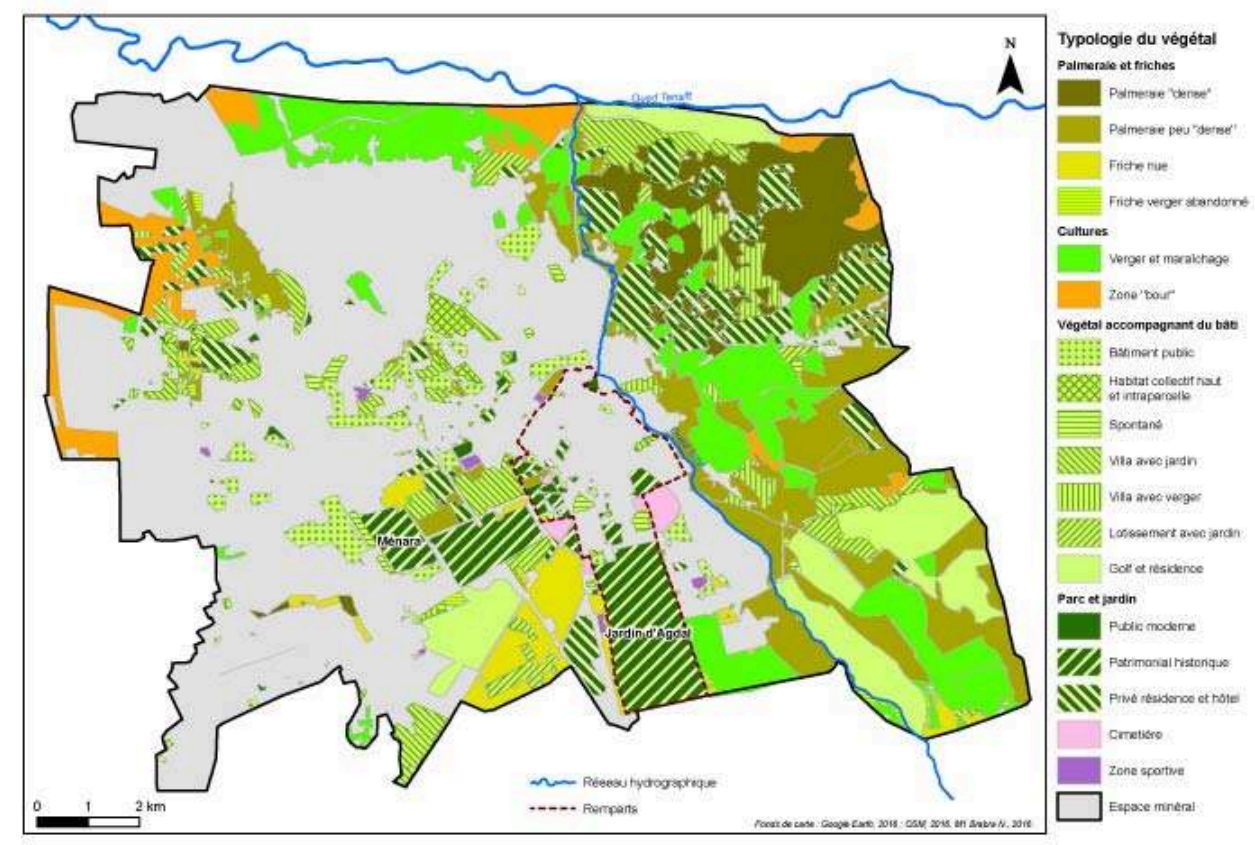

- Le premier groupe (4 classes) est constitué de la palmeraie subdivisée en « dense » et «peu dense ». Un sous-ensemble de friches nues et de vergers abandonnés a été inclus dans ce groupe.

- Le deuxième groupe (2 classes) correspond aux zones de cultures fonctionnelles avec du maraîchage et des vergers dominés initialement par les oliveraies et des agrumes. Cependant en sont exclus les anciens jardins d'Agdal ou de la Ménara qui, bien que dédiés à la production agricole, sont avant tout considérés comme des jardins patrimoniaux.

- Le troisième groupe (7 classes) comprend le végétal qui accompagne du bâti, constitué de sous-ensembles: du végétal associé aux habitats collectifs (petits jardins au pied des immeubles ou végétal spontané) ; des jardins de villas de luxe et de lotissements ; des jardins associés aux infrastructures touristiques. La quantification de ces sous-groupes est imprécise car elle inclut les bâtiments.

- Le quatrième groupe ( 5 classes) est composé des parcs et jardins publics datant de la période coloniale ou associés à la ville coloniale, les anciens jardins patrimoniaux et historiques, dont certains correspondent à des vergers en exploitation (Agdal, Ménara...), ainsi que les cimetières et terrains de sport.

11 Cette typologie reste encore à affiner, et la quantification des surfaces de chaque type de végétal à l'échelle des différents quartiers est encore très imprécise car nécessite une couverture de photographie aérienne à grande échelle. Elle n'inclut pas, notamment, les surfaces couvertes par les arbres d'alignement, difficiles à visualiser sur les images satellites et pas encore cartographiés sur le terrain. Cependant, les superficies qu'ils couvrent sont modestes. 
12 La dynamique d'évolution rétrospective de ce végétal a ensuite été mise en évidence à partir de l'analyse de documents d'archives, pour l'essentiel du Centre des archives diplomatiques de Nantes. Ce sont notamment les mémoires de stages des contrôleurs civils stagiaires et des élèves de l'École nationale d'administration des fonds "protectorat français au Maroc", mais également les fonds iconographiques et cartographiques mis à disposition par la Bibliothèque nationale de France (notamment le fonds Henri Prost). Les seuls travaux qui traitent en partie du végétal et de son évolution à Marrakech restent très généraux, limités à certains jardins et dominés souvent par la question de l'évolution de la palmeraie (El Faïz, 1996, 2000 et 2002; Pordany-Horvath, 2004).

13 Cette approche historique de la présence du végétal en ville vise à mettre en évidence l'emplacement et l'évolution des espaces végétalisés et les fonctions qui leur étaient attribuées en replaçant chronologiquement la création et les transformations des espaces verts. Elle cherche aussi à identifier, aux différentes périodes précoloniale, coloniale et post-coloniale, les intentions des services techniques, des urbanistes ou des habitants lors de l'implantation du végétal en ville et, dans une certaine mesure, la perception qu'en ont les édiles et les voyageurs.

\section{Résultats et discussion}

\section{Marrakech une cité-jardin : historique}

Dès sa création en 1071 (an 463 de l'hégire) par la dynastie des Almoravides, les fondateurs de Marrakech ont d'abord cherché à mettre en place un "écosystème " urbain, lieu d'actions, interactions et retroactions complexes entre les différentes composantes urbanistiques, socioculturelles et environnementales de ce système, basé sur une autonomie de ressources assurant aux nouveaux maîtres de cette plaine, les Almoravides, territoire historique de la tribu Sanhadja, une sécurité alimentaire.

C'est dans ce contexte conflictuel que les nouveaux habitants ont disposé de terrains relativement vastes leur permettant de pratiquer de l'agriculture et même de l'élevage à l'intérieur de la cité. "Chaque palais a ses propres jardins, son propre bain, ses propres ressources en eau, [...], le propriétaire ferme ses portes pour toute sa famille, ses biens et ses animaux, il ne sort pas de chez lui pour acheter ce dont il a besoin... » (Aârabi, 201411), Le développement d'une agriculture urbaine posant le problème de la ressource en eau dans ce contexte aride, de nombreux puits ont donc été creusés. Cependant, face au développement de la ville devenue capitale de l'empire, c'est la construction d'un système de galeries souterraines (les khettaras), assurant le transfert de l'eau du piémont de l'Atlas vers la ville (El Faïz, 2002), qui constitue le vrai changement dans la mise en place d'un cadre urbain dominé par le végétal à l'intérieur de la ville et dans son environnement immédiat, c'est-à-dire la palmeraie. Cette technique a permis à la ville de disposer d'une ressource abondante disponible toute l'année et de se libérer du contrôle des tribus hostiles du piémont.

Évoluant dans un contexte sociopolitique très conflictuel et des conditions bioclimatiques semi-arides favorisant des paysages qualifiés de " désertiques ", la villeoasis suscitera un imaginaire paysager dont la palmeraie, la cité ocre et le Haut-Atlas en arrière plan sont les éléments structurants. Il est intéressant de noter que dans ces représentations qui perdurent, le qualificatif de désertique, souvent évoqué par les 
voyageurs occidentaux, ne l'est pas par les voyageurs arabo-musulmans. Ainsi pour Jean Claude Nicolas Forestier (1913) : «Rien n'est plus saisissant que le spectacle qui s'offre à la vue lorsqu'en arrivant de Casablanca, on aperçoit tout d'un coup, à quelques kilomètres avant Marrakech, au milieu d'une grande plaine d'aspect désertique barrée au sud par la ligne bleu et blanc de l'Atlas couvert de neige, le vert imprévu, abondant, le vert frais, reposant, d'une immense oasis, dans laquelle se dissimule presque la vieille ville berbère. »Cette description et ce ressenti exprimé par Forestier qui effectue son premier voyage au Maroc en 1913 sont récurrents dans de nombreux récits de voyageurs depuis le XVIII ${ }^{\mathrm{e}}$ siècle.

«Les jardins de palmiers et d'oliviers qui font presque de tous les côtés une seconde enceinte à la ville s'approchent par endroit jusqu'à toucher la muraille, et les palmiers penchent leurs têtes sur les créneaux délabrés. Mais le plus souvent, au pied des murs s'étend un terrain vague, d'un extraordinaire aspect. Partout des trous béants, dont la terre est rejetée sur les bords, des excavations profondes qui ont servi naguère à creuser les galeries souterraines par où arrivent de très loin les eaux de source à Marrakech. Un grand nombre de ces aqueducs centenaires se sont effondrés au cours du temps [...]. Ailleurs plus de palmiers, plus d'oliviers, plus rien. Rien que l'immensité nue, verdoyante au printemps, complètement brulée l'été, d'où l'on voit accourir de loin les longs chapelets des puits qui apportent la fraîcheur à la ville et aux jardins, tandis que là-bas, à l'horizon, l'Atlas déroule sans limites des hautes vagues immobiles, resplendissantes de leur écume neigeuse qui ne s'écroule jamais sur la plaine ...» (Tharaud, 2012.)

18 Ce contexte paysager de cité au milieu d'une vaste plaine aride, entourée d'une oasis et dominée par une haute montagne, a suscité un imaginaire fécond et structuré dès les premières "découvertes" de la ville par les Européens. Ces représentations sont aujourd'hui toujours vivaces. La montagne qui la domine, source vitale d'alimentation en eau, est aussi à l'origine de l'instabilité et parfois du déclin de cette ville. Chaque fois que la cité a perdu son statut de capitale au profit d'autres villes, les tribus avoisinantes ont souvent cherché à la détruire. Son déclin se traduisait alors par une régression démographique et l'abandon des jardins et des vergers. À l'aube de la colonisation, Marrakech était ainsi devenue une cité "fantôme", et selon Washington (1830) «plusieurs quartiers sont disparus...L'herbe qui y croit forme un frappant contraste avec les débris de muraille qu'on y distingue » (cité par Wilbaux, 2001).

\section{Dynamique du végétal : une régression qualitative et quantitative}

19 À partir de la période coloniale, la dynamique d'évolution du végétal prend des formes différentes à l'intérieur et à l'extérieur des remparts de la ville.

\section{Dynamique du végétal intra-muros}

20 Le rapport de force qu'a entretenu depuis l'origine la ville de Marrakech avec les tribus avoisinantes est la cause principale qui justifie le développement des vastes jardins intra-muros. Guy Dugas (1996) décrit qu'«à mesure qu'on s'éloigne de l'enchevêtrement des ruelles qui forment le cœur de la cité, des chemins plus larges s'en vont entre des murs de vergers, derrière lesquels les arbres et les plantes sont soustraits aux regards aussi jalousement que les femmes ». Ces jardins constituaient une ceinture verte longeant les remparts et encadrant la partie urbanisée comme en 
témoigne la toponymie de certaines rues nommées du nom des anciens jardins présents comme Arset (jardin) El Houta, Arset Moulay Bouazza, etc. On percevait encore en 1918 (figure 4) cette organisation avec deux enveloppes végétales; celle des jardins et des vergers intra-muros, et l'enveloppe extra-muros dominée par la palmeraie et les vergers où commence à se développer à cette date le premier front urbain de la nouvelle ville coloniale au nord-ouest (quartier de Guéliz).

Figure 4. La ville de Marrakech en 1918 (d'après le Service topographique du Maroc)

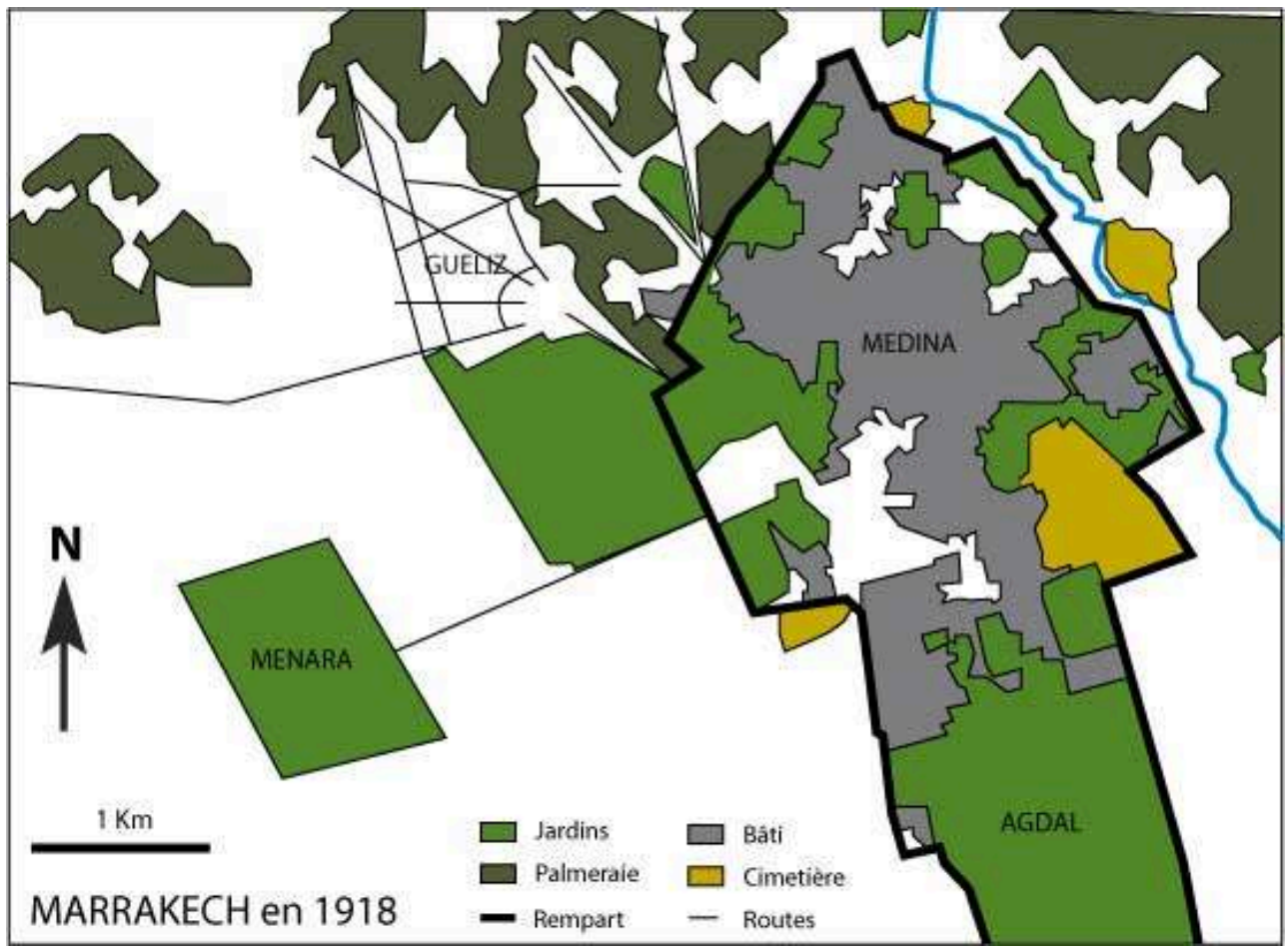

Cette agriculture à l'intérieur des remparts assurait à la ville une certaine autonomie alimentaire surtout en période de siège. À la veille de la colonisation, le végétal, qui couvrait plus de $80 \%$ de la superficie de la médina (El Faïz, 2000), était constitué d'un système de jardins royaux ou de notables, les Agdals (jardins des sultans et notables de la ville associés à de grandes demeures, palais, ou maisons, situés sur les marges de la médina ou à l'intérieur) et de simples jardins longeant les remparts. Comme évoqué précédemment, la principale fonction de tous ces jardins est d'abord alimentaire. Mais ils constituent aussi "l'espace vert» de la cité, lieu de N'Ezha (pique-nique) hebdomadaire ou pendant certaines fêtes à caractère religieux. Dans son premier rapport sur la cité, Jean Claude Nicolas Forestier (1913) évoque ainsi la richesse et l'importance de ces jardins pour la population de la ville.

Par la suite, le maréchal Lyautey, premier résident général du protectorat français au Maroc en 1912, est à l'origine de l'évolution des paysages de cette cité et de la mise en place d'une dichotomie urbaine et paysagère qui a longtemps structuré la morphologie de Marrakech et conditionné en partie son évolution future. Le bilan que fait le maréchal de l'expérience coloniale à la française en Algérie est à l'origine de sa théorisation de la séparation spatiale de la société européenne et celle qualifiée d'indigène. Le concept de la séparation spatiale ne signifie pas rupture, mais plutôt la mise en place d'un processus d'interpénétration sélective entre deux modèles 
différents. Il fallait, selon Lyautey, conserver l'existant sans remettre en cause ses fondamentaux, surtout culturels. "L'expérience de trop de villes algériennes était là pour nous l'enseigner. Il était donc bien simple, puisque l'on devait en sortir, de commencer par se mettre dehors. C'est de là qu'est partie notre conception initiale. Toucher le moins possible aux villes indigènes. Aménager à leurs abords, sur les vastes espaces encore libres, la ville européenne, suivant un plan réalisant les conditions les plus modernes. » (Lyautey cité par Mouline, 1987.)

Comme le montrent les premiers plans de Henri Prost, architecte urbaniste qui a conçu la nouvelle ville de Marrakech, la médina de Marrakech n'est pas censée subir de transformations majeures, sans doute sur les recommandations de Jean Claude Nicolas Forestier. Elle est seulement retouchée par quelques aménagements sous forme de voies de communication ou de bâtiments marquant une forme de continuité entre les deux univers.

Une des principales conséquences de cette politique de préservation de la médina est le développement d'une vision statique et figée de la société indigène, cloîtrée dans son espace bien délimité par des remparts, dépourvue de dynamique et vivant hors de toutes influences extérieures. Cependant, face à la croissance démographique de la population de la médina, amplifiée par l'exode rural, l'extension des espaces bâtis, contrainte dans les remparts, s'est faite aux dépens des jardins. L'autre effet a été l'apparition des premiers quartiers informels (les Douars) autour de la ville, essentiellement composés d'un bâti dense et dépourvus de végétation. Vers la fin de la période coloniale, et à l'exception des jardins royaux et quelques Agdals appartenant aux grandes familles de notables, la majorité des jardins situés à l'intérieur des remparts avaient disparu, mettant fin au modèle de la « ville jardinée ».

\section{Dynamique extra-muros : la ville coloniale, premier « front urbain »}

Hors des remparts, la principale transformation pendant la colonisation fut la création d'une nouvelle ville moderne concrétisant la vision de Lyautey. Cette ville fait face à la médina en occupant son flanc ouest aux dépens d'une partie de sa palmeraie. Il s'agit du premier front urbain, lisible sur les séries de cartes produites dès le début de la colonisation (figure 4), ouvert au sein de ce couvert végétal qualifié d'oasis. Sur le modèle d'une cité jardin (El Faïz, 2000 et 2002), cette ville nouvelle est composée de quartiers administratifs et résidentiels où le végétal dans l'espace public est un élément participant à sa structuration, à sa morphologie et à son organisation. C'est ce caractère public qui fait la différence par rapport à la cité-jardin version " modèle médina ", où le végétal est privé avant tout, et qui est dépourvue d'espaces verts dans l'espace public. La nouvelle ville est composée de deux quartiers : le Guéliz et l'Hivernage (figure 2). Le Guéliz est dominé par un habitat dense associé à une activité commerciale et administrative. En revanche, l'Hivernage est un quartier exclusivement résidentiel (résidences privées et touristiques) réservé à la détente et aux loisirs. C'est un lieu de villégiature destiné aux classes supérieures. C'est dans ce quartier que vont s'implanter les premiers équipements destinés aux activités touristiques (casino...) qui se développeront après l'indépendance, faisant de ce quartier une zone touristique par excellence.

L'esquisse du plan de plantations du quartier de l'Hivernage conçu par Prost (figure 5), qui assure la liaison entre la médina et le Guéliz, montre clairement son caractère de quartier-jardin. Sa conception intègre parfaitement les éléments structurant l'identité 
paysagère de la ville avec « un tracé courbe propre à la flânerie, prenant ses vues sur les remparts, la Koutoubia, les sommets neigeux de l'Atlas » (Forestier, 1913). Quartier destiné à une clientèle aisée et aux activités touristiques, les fonctions du végétal y sont donc désormais avant tout esthétiques. Il vise aussi à créer un microclimat agréable atténuant les fortes chaleurs par l'ombre et l'effet de l'évapotranspiration. Associés à un système d'arcade le long des rues orientées de manière à favoriser les flux d'air rafraichissants dans le quartier de Guéliz, les arbres d'alignement (Citrus aurantium, Ficus...) étaient totalement intégrés dans la réflexion urbanistique de Forestier reprise par Prost. L'ambiance urbaine ainsi créée visait à favoriser une qualité de vie pour les populations européennes de ces quartiers.

Figure 5 : Quartier de l'Hivernage

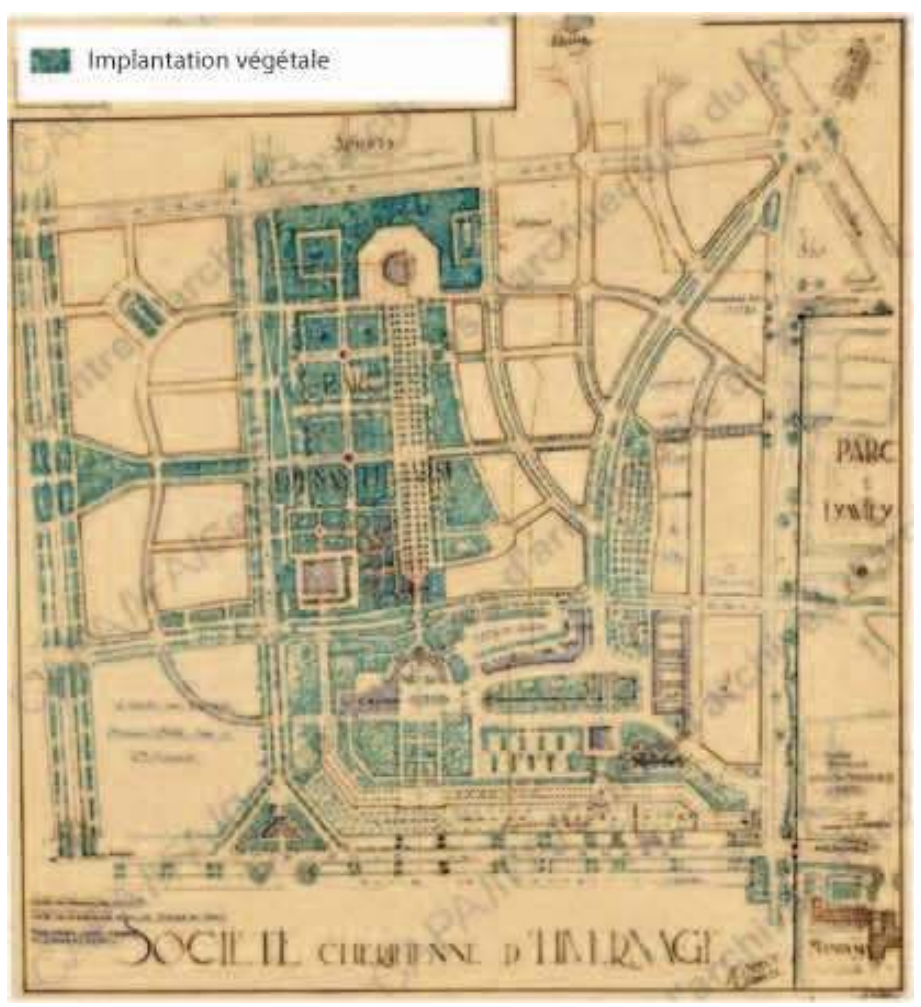

Aménagement du secteur d'hivernage à Marrakech : plan d'aménagement (constructions et plantations), 11 octobre 1939).

Source : Fonds Prost Henri, 1929-1933, Centre d'archives d'architecture du xx siècle, http:// archiwebture.citechaillot.fr/fonds/FRAPN02_PROST/inventaire/objet-21564.

À travers son organisation et la nature de ses fonctions, la ville nouvelle traduit ce qu'est et doit être une ville coloniale : une géométrie parfaite associant fonctionnalité et efficacité. Cela reflète aussi ce que doit être une ville moderne au sens occidental.

\section{Dynamique extra-muros : une cité-jardin devenue « minérale » après l'indépendance}

Depuis l'indépendance en 1956, la croissance démographique de la ville de Marrakech s'est traduite par une urbanisation croissante impactant profondément les paysages de la médina et son environnement immédiat. Si les nouveaux quartiers de la ville coloniale ont constitué la première rupture dans le modèle urbain marocain structuré 
par les villes impériales, le processus d'urbanisation moderne très rapide qui a suivi a bouleversé les paysages urbains du Maroc (Mohaine, 2017). La ville de Marrakech n'a pas échappé à cette tendance dont la surface urbanisée a été démultipliée depuis l'indépendance.

La croissance démographique de la ville a débuté depuis la période coloniale et elle constitue le principal facteur de son extension effrénée, que ce soit à l'intérieur de la médina (densification) ou au-delà des remparts. La ville comptait ainsi 439738 habitants en 1982 et 974021 habitants en 2014, date du dernier recensement (RGPH), même si le taux d'accroissement annuel de la population montre un ralentissement sur cette période, passant de 4,3\% entre 1982 et 1992 à 2,2\% entre 1994 à 2004 . Les différents plans élaborés par l'agence urbaine de Marrakech n'ont pas réussi à réguler ni à accompagner l'évolution de la ville (un nouveau plan est en cours d'élaboration).

Cette dynamique souvent qualifiée "d'anarchique » ou non maîtrisée a généré des paysages qui ne font référence à aucun des deux modèles préexistants de la médina ou de la ville coloniale. Il s'agit d'une deuxième rupture dans le développement de cette cité-oasis, impactant profondément le végétal.

31 Cette extension multidirectionnelle n'a épargné aucun des espaces qui la composent, pas même sa palmeraie qui jouissait pourtant du statut théoriquement protecteur de patrimoine historique depuis 1945 (Dahir 21 juillet 1945). Très convoitée pour son cadre paysager, la palmeraie est grignotée par des villas, des hôtels et autres infrastructures touristiques (golfs, etc.). Si en 2016 les surfaces végétalisées représentaient 51,9\% de la surface du quartier Annakhil de la palmeraie ( $28,9 \%$ de la zone d'étude), une bonne partie de ce végétal est aujourd'hui intégré à l'intérieur des enceintes de ces espaces privés (figure 3).

32 Aujourd'hui, la minéralisation de la ville est en progression constante, une nouvelle ville (Tamansourt au nord-ouest de Marrakech) est en train de prendre forme, de très nombreux complexes immobiliers voient le jour le long de tous les axes autour de la ville et d'autres quartiers résidentiels sont déjà programmés. Ce processus de minéralisation ne concerne pas seulement les nouveaux quartiers, il s'étend à la ville coloniale où la densification, voulue par les autorités de la ville, s'est traduite par le développement d'immeubles de cinq étages aux dépens d'anciennes villas avec jardins (figure 6), particulièrement dans le quartier de Guéliz, très recherché par les investisseurs immobiliers, où elles ont quasiment disparu.

Cette transformation est en train d'impacter aussi le quartier-jardin de Forestier: l'Hivernage. Les nouvelles constructions ne conservent aucun espace végétalisé et il est fréquent de voir certains propriétaires couper les arbres d'alignement situés dans l'espace public jouxtant leur immeuble. La couverture végétale de ces deux quartiers n'était plus que de 5,3\% (correspondant à $3 \%$ de la couverture végétale de la ville) en 2016 (figures 2 et 3 ). 
Figure 6. Densification de la ville coloniale dans le quartier de Guéliz

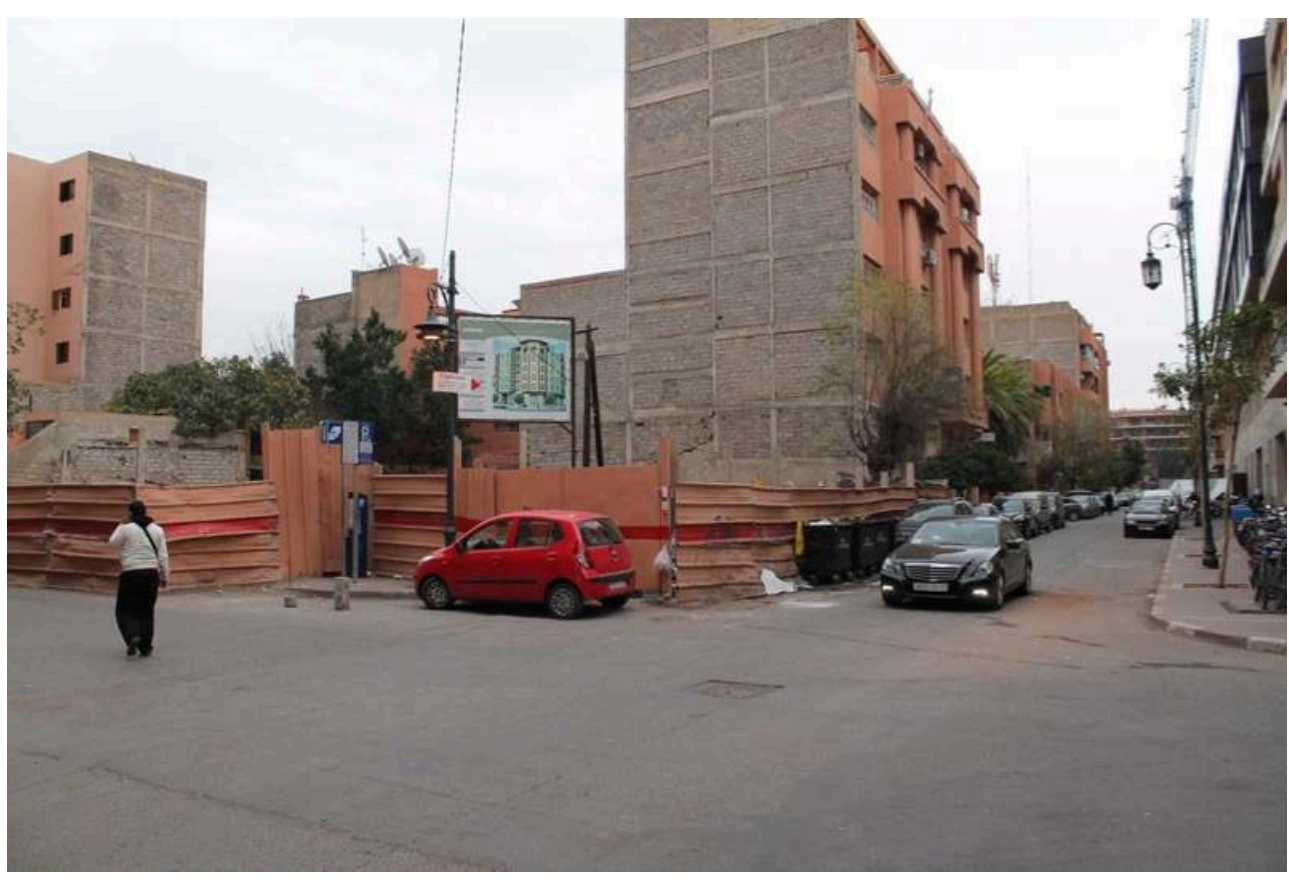

Jadis cité-jardin, Marrakech n'offrait plus que $2 \mathrm{~m}^{2}$ d'espace vert à ses habitants au début $\mathrm{du} \mathrm{xx}^{\mathrm{e}}$ siècle contre $60 \mathrm{~m} 2$ au début du siècle dernier (El Faïz, 2000). Si l'accroissement démographique est un facteur déterminant dans les transformations en cours, elles sont inéluctables dans un pays dont la jeunesse (0-25 ans) représente plus de $50 \%$ de la population. Il faut donc aussi questionner les formes de gestion et les modalités de planification mises en place depuis l'indépendance. Dans les nombreux nouveaux quartiers qui ont vu le jour, le végétal n'est pas pensé comme une composante de l'espace public, ni en relation avec le type de bâti. C'est le cas des quartiers construits dans les années 1970 et 1980 au nord de la ville (Daoudiate, etc.), accueillant plutôt une classe moyenne, où le couvert végétal ne représente que $2,2 \%$ de la surface de ces quartiers en 2016 (figures 2 et 3) et correspond à 1,2\% de la surface végétale de Marrakech. Le quartier populaire plus ancien de Sidi Youssef Ben Ali (SYBA), construit dans les années 1950 et 1960, présente les mêmes taux de végétalisation (figures 2 et 3 ) avec $2,4 \%$ de couverture végétale ( $1,3 \%$ de la ville). Si les quartiers les plus récents, construits à partir des années 1990 dans l'ouest de la ville, sont globalement un peu plus végétalisés avec une couverture végétale de $13 \%$, cela masque en réalité une grande disparité entre les parties populaires quasiment sans végétal (figure 7) et les quelques quartiers de villas avec jardins (figures 2 et 3 ). 
Figure 7. Développement de nouveaux quartiers caractérisés par l'absence totale de végétal dans l'espace public

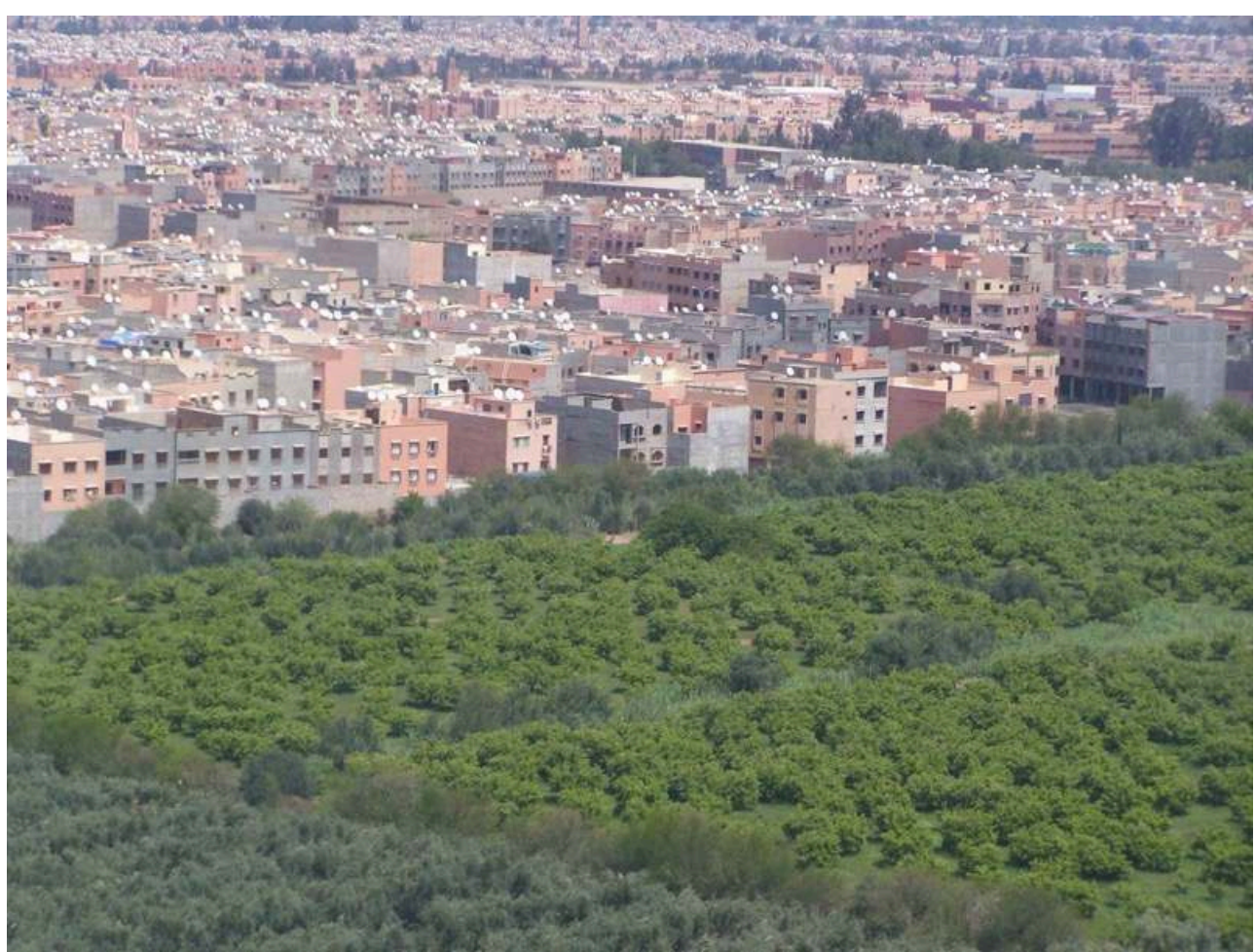

Front urbain de l'ouest de Marrakech.

On peut aussi s'interroger sur l'absence de végétalisation des nouveaux quartiers et le délaissement des arbres d'alignement de la période coloniale dans les anciens quartiers qui traduit une rupture culturelle. Concerne-t-elle les habitants ou les acteurs de la planification et les urbanistes? En effet, le désir de végétal au sein de la population est une réalité qui s'exprime à travers plusieurs types d'actions visibles dans l'espace public comme son appropriation par la plantation ou la mise en pot d'arbres et de buissons sur les trottoirs devant les maisons (figure 8). 
Figures 8. Végétalisation et appropriation de l'espace public par les habitants dans deux quartiers à profil social différent

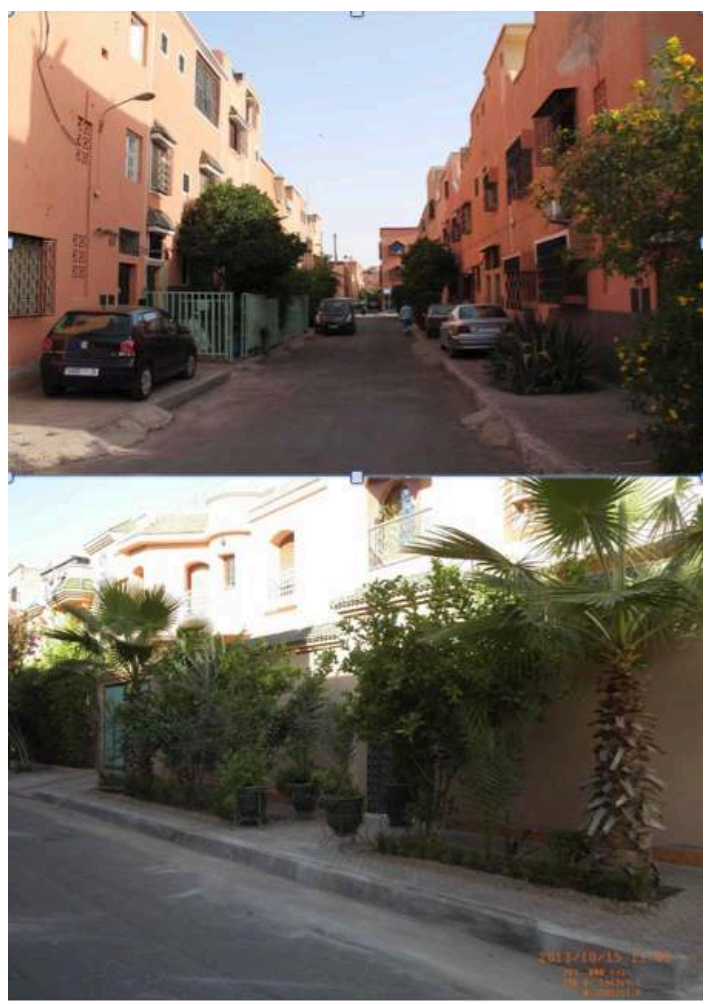

Une rue dans un lotissement (haut) où les trottoirs ont été plantés d'arbres et d'arbustes et souvent grillagés, et un quartier de villas (bas) où un minijardin d'embellissement de la façade et de l'entrée de la villa empiète sur le trottoir.

\section{La végétalisation : une variable de discrimination sociospatiale}

Les vergers et les jardins qui constituaient l'enveloppe verdoyante de la ville sont remplacés par des quartiers résidentiels dont la végétalisation est fonction du type de quartier et de la population qui l'occupe.

L'analyse des différents quartiers de la ville montre d'importants contrastes du degré de végétalisation, discriminant notamment les quartiers pauvres ou de classe moyenne et les quartiers riches et quartiers touristiques (figures 3, 7 et 8).

Dans les quartiers à forte densité dominés par le logement social, et souvent associés à une population pauvre ou modeste, le végétal est quasiment inexistant. À Sidi Youssef Ben Ali ou dans les quartiers nord de Marrakech construits dans les années 1970 et 1980 , les taux de végétalisation sont de $2,4 \%$ et $2,2 \%$ et correspondent à un végétal surtout associé à des bâtiments publics (lycées, etc.) et à des zones sportives ou inclus dans des résidences et lotissements privés. Le terrain a montré que quelques associations de quartier se mobilisent parfois pour tenter d'introduire un peu de verdure en pot le long des rues et d'inciter les habitants à en assurer l'entretien.

39 À l'opposé, là où dominent les maisons individuelles de lotissement, ce sont les habitants qui initient la végétalisation de leurs rues. Elle prend alors la forme d'une appropriation de l'espace public. Ayant bâti la totalité de leur parcelle sans prévoir de 
jardin, ils plantent des arbres et arbustes (orangers amers, hibiscus...) et plantes grimpantes (bougainvilliers, jasmins...) le long des trottoirs avoisinants leurs habitations (figure 8). Certains se sont créé ainsi de vrais jardins en introduisant de nouvelles espèces et en transformant une partie en garage pour voiture.

Dans les quartiers de villas occupés par une population aisée, dans la palmeraie ou certains quartiers récents implantés depuis les années 1990, la végétalisation rappelle celle du quartier de l'Hivernage. Disposant de revenus élevés, les habitants y prennent en charge financièrement l'entretien et la gestion de leurs jardins privés ainsi que des espaces verts publics constitués d'arbres d'alignement.

La gestion du végétal dans l'espace public par la municipalité (conception, plantations, entretien...) est concentrée sur les grandes artères (Washingtonia, Phoenix dactilifera, Citrus aurantium...), certaines places et les parcs et jardins très fréquentés par la population et surtout par les touristes (El Harti, Aarsat Moulay Abdeslam, etc.). Cependant, dans de nombreux quartiers, c'est souvent la population qui tente de végétaliser son cadre de vie. Il ne s'agit nullement d'une vraie politique de transfert de compétences de la part de la municipalité vers la population, mais plutôt d'initiatives observées sur le terrain, émanant et relevant d'associations ou de collectifs de quartiers qui décident d'engager la végétalisation de leurs rues pour en améliorer la qualité. Il n'est pas rare alors que la population recrute et assure le salaire des jardiniers en demandant une cotisation aux habitants, surtout dans les quartiers d'habitations collectives.

\section{Conclusion}

Jadis cité-jardin ou cité-oasis, l'identité paysagère de la ville de Marrakech est indissociable du végétal. Cette identité, qui incluait la médina durant dix siècles, avait trouvé une certaine continuité dans la ville coloniale, même si on a bien deux modèles de cité-jardin dont les fonctions et les formes du végétal diffèrent, surtout au niveau de l'espace public. La ville coloniale, qui constitue une rupture du point de vue urbanistique, avait cependant bien su intégrer dans son organisation et son fonctionnement les anciens jardins (Jnan El Harti, Aarsat Moulay Abdeslam, etc.) comme jardins publics ou jardins historiques à valeur patrimoniale. L'attribution de nouvelles fonctions à ces espaces a certainement permis d'en préserver quelques-uns jusqu'à présent.

43 La plupart des nouveaux quartiers de classes moyennes et modestes ne disposent d'aucun espace vert, d'aucun alignement d'arbres. Le végétal est devenu une variable discriminante entre quartiers pauvres et quartiers riches. Pour les décideurs, seuls les grandes avenues et quelques places et jardins publics méritent intérêt, car ils participent à préserver l'image de "cité verte» véhiculée par la communication destinée surtout aux touristes.

Le processus de minéralisation des paysages de la ville est une réalité, elle à presque quadruplé sa superficie urbanisée aux dépens de ses vergers et même de sa palmeraie qui est devenue un simple décor pour complexes touristiques et villas de haut standing. La communication touristique et celle des projets immobiliers de cette partie ouest de Marrakech vantent ainsi ce cadre paysager, et les actions de reboisement menées par le plan de sauvegarde de la palmeraie, porté par la Fondation Mohammed VI pour la 
protection de l'environnement, montrent clairement que c'est la conservation du palmier qui est privilégiée au détriment de la palmeraie.

L'absence du végétal dans la planification des nouveaux quartiers essentiellement composés de classes moyennes et modestes est une réalité que la population tente d'atténuer en improvisant des petits espaces verts ou en végétalisant leurs rues.

La démographie et l'exode rural constitueront toujours un défi pour cette cité réputée pour son ouverture sur la campagne dont est originaire la majorité des nouveaux habitants. Ces derniers continueront à affluer vers la cité, synonyme de "progrès social ».

La question qui se pose, et qui constitue aussi un enjeu, est de comprendre pourquoi cette cité ne cherche pas à s'appuyer sur son double héritage de la médina et de la ville coloniale afin de repenser la place du végétal dans son développement et à l'échelle de tous les quartiers. Le désir de végétal est là. Même s'il est souvent un élément d'appropriation de l'espace public, il peut être aussi une forme de «socialisation » de ce dernier.

\section{BIBLIOGRAPHIE}

Aârabi, AK., مراكش التآسيس و التسمية دراسة لنصوص تاريخية, Marrakech : Fondation et nomination, étude de textes historiques, Marrakech, Fondation Horizons, 2014.

Bennani, M., « Le rôle fondateur du paysage dans la création des villes coloniales marocaines : Rabat et Marrakech, deux exemples de villes-jardins ", Projets de paysage, $n^{\circ} 7$, janvier 2012, URL : https://www.projetsdepaysage.fr/fr/ le_r_le_fondateur_du_paysage_dans_la_cr_ation_des_villes_coloniales_marocaines Boujrouf, S., « Tourisme et aménagement du territoire au Maroc : quels agencements ? ", Téoros, 24-1, 2005, URL : http://teoros.revues.org/1490.

Boubkraoui, A., « La palmeraie de Marrakech aujourd'hui. Aspects et causes de la dégradation », actes des deuxièmes journées de l'arbre, Marrakech, avril 1994.

Chevrillon, A., Marrakech dans les palmes, Paris, Edisud, 2010.

Coslado, E., «Itinéraires d'individuation dans la périphérie de Marrakech : mobilités résidentielles et recompositions territoriales d'habitants de classes moyennes ", Les Cahiers d'EMAM, n ${ }^{\circ}$ 16, juillet 2008, p. 37-50.

Deverdun, G., Marrakech des origines à 1912, Rabat, Éditions techniques nord-africaines, 2 vol., 1959.

Doutté, E., Merrâkech, Paris, Comité du Maroc, 1905.

Dugas, G., Maroc, Les villes impériales, Paris, Omnibus, 1996.

El Faïz, M., Les Jardins historiques de Marrakech : mémoire écologique d'une ville impériale, Florence, Edifir, 1996. 
El Faïz, M., Les Jardins de Marrakech, Arles, Actes sud, 2000.

El Faïz, M., Marrakech : patrimoine en péril, Arles, Actes sud/Eddif, 2002.

El Houmaizi, M.A., Oihabi, A., Saaidi, M., « La palmeraie de Marrakech : ses contraintes et ses atouts ", Sécheresse, vol. 9, nº 2, 1998, p. 163-166.

Fairchild Ruggles, D., Islamic Gardens and Landscapes, Philadelphie, University of Pennsylvania Press, 2008.

Forestier, J. C. N., « Rapport des réserves à constituer au dedans et aux abords des villes capitales du Maroc », Grandes Villes et Systèmes de parcs (1913), présenté par Leclerc, B. et Tarrago, S., Paris, Norma/IFA, 1997.

Forestier, J. C. N., Grandes Villes et Systèmes de parcs, présenté par Leclerc, B. et Tarrago, S., Paris, Norma/IFA, 1997.

L'Africain, J.-L. (Al Hassan al-Wazzan), Description de l'Afrique, traduit de l'italien par Epaulard, A., Paris, Adrien Maisonneuve, 1956.

Mohaine, A., La Géographie et l'Aménagement au Maroc. Regards croisés, Casablanca, Afrique Orient, 2017.

Mouline, S., "Architecture métissée et patrimoine », Old cultures in new worlds, 8th Icomos General Assembly and International Symposium, Washington, Icomos, 1987, p. 715-722.

Pordany-Horvath, C., « La genèse des jardins en islam et les jardins historiques de Marrakech ", workshop de la CUPEUM Marrakech 2004 « La Palmeraie de Marrakech - un paysage périurbain », chaire Unesco paysage et environnement, 2004, URL : www.unesco-paysage.umontreal.ca.

Taïbi, A. N., El Hannani, M., « Marrakech, la ville-oasis ? », dans « 8e Maghreb créateurs », Angers, Kalima Association culturelle Franco-Arabe, 7 avril 2015.

Tharaud, J. et Tharaud, J., Marrakech ou les Seigneurs de l'Atlas (1920), Casablanca, Dar el Aman, 2012.

Wilbaux, Q., La Médina de Marrakech. Formation des espaces urbains d'une ancienne capitale du Maroc, Paris, L'Harmattan, 2001, 381 p.

\section{NOTES}

1. Aârabi citant Al kachladi soubh al aacha fi sinaati Al Incha, t. 5, Le Caire, 1915. Citation traduite par Mustapha El Hannani.

\section{RÉSUMÉS}

Marrakech s'est développée sur le modèle classique de la cité-jardin insérée dans une oasis. Dans l'iconographie et les descriptions d'écrivains et voyageurs, Marrakech est indissociable de sa palmeraie et du végétal, élément identitaire fort et cadre paysager qu'elle a su valoriser pour le tourisme. Cette image est de plus en plus déconnectée de la réalité, la cité-jardin et l'oasis 
s'estompent devant l'extension et la densification de la ville. La palmeraie et les arbres d'alignement reculent face à l'urbanisation, au développement des golfs, des complexes touristiques et des villas, cela depuis la période coloniale et à un rythme accéléré ces dernières décennies. La composition de cet héritage végétal reflète les différentes influences culturelles qui ont façonné l'identité actuelle de la ville. La palette de végétaux révèle un changement qualitatif dans l'évolution des paysages corrélé avec celui des goûts et choix d'une classe sociale et d'une population remettant en cause cette identité, basée historiquement sur un équilibre entre végétal et minéral. La gentrification s'accompagne d'un abandon des fonctions nourricières au profit de fonctions récréatives et paysagères.

Marrakesh was developed based on the classic model of the garden-city imbedded in an oasis. In the iconography and descriptions of writers and travellers, Marrakesh is indissociable from its palm groves and plants which confer a strong identity to a landscape the city has promoted for tourism. This image has increasingly become disconnected from reality, the garden-city and oasis image is fading away in the face of the extension and densification of the city. The palm groves and rows of trees have been retreating with encroaching urbanisation, golf courses, tourist complexes and villas, ever since the colonial period and increasingly in recent decades. The composition of this plant heritage is a reflection of various cultural influences which have shaped the current identity of the city. The palette of plant species reveals a qualitative change in landscapes correlated to changes in the tastes and choices of a social class and a population which questions this identity, historically based on a balance between plant and mineral elements. Gentrification has led to food production being abandoned to the benefit of recreational and aesthetic landscape functions.

\section{INDEX}

Mots-clés : cité-jardin, minéralisation, discrimination, rupture culturelle

Keywords : garden-city, mineralisation, discrimination, cultural disruption

\section{AUTEURS}

\section{MUSTAPHA EL HANNANI}

Mustapha El Hannani, géographe, maître de conférences, ESO-Angers UMR 6590 CNRS, université d'Angers, UBL.

mustapha.elhannani[at]univ-angers[dot]fr

\section{AUDE NUSCIA TAÏBI}

Aude Nuscia Taïbi, géographe, maître de conférences HDR, LETG-Angers UMR 6554 CNRS, université d'Angers, UBL.

nucia.taibi[at]univ-angers[dot]fr

\section{NAIIMA BRABRA}

Naïma Brabra, géographe, étudiante en master 2 PUSM, ESO-Angers UMR 6590 CNRS, université d'Angers, UBL.

naima.brabra[at]etud.univ-angers[dot]fr 


\section{SIGRID GIFFON}

Sigrid Giffon, cartographe, assistante ingénieure, LETG-Angers UMR 6554 CNRS, université d'Angers, UBL.

sigrid.giffon[at]univ-angers[dot]fr 